\title{
DUELOS FAMILIARES O COLECTIVOS. DISEÑO DE UNA ESCALA DE ACTITUDES HACIA LOS NIÑOS EN PROCESOS DE DUELO
}

\section{FAMILY OR COLLECTIVE GRIEF. DESIGN OF A SCALE OF ATTITUDES TOWARD CHILDREN IN MOURNING PROCESS}

\author{
Marta Villacieros Durbán, José Carlos Bermejo Higuera y \\ Marisa Magaña Loarte
}

Centro de Humanización de la Salud, Tres Cantos, Madrid, España

Cómo referenciar este artículo/How to reference this article:

Villacieros Durbán, M., Bermejo Higuera, J. C., y Magaña Loarte, M. (2016). Duelos Familiares o Colectivos. Diseño de una Escala de Actitudes hacia los Niños en Procesos de Duelo. Revista de Psicoterapia, 27(104), 167-179.

\section{Resumen}

Objetivo: Diseño y validación de una escala breve de actitudes de los adultos hacia los niños en los procesos de duelo familiares o colectivos. Método: Se obtuvieron 227 cuestionarios válidos, 86\% (191) mujeres y $14 \%$ (31) hombres, con una edad media de 41 años. $A$ partir de 50 items evaluando las actitudes de los adultos hacia los niños en situación de duelo, se realizó un análisis de normalidad de las variables, de fiabilidad (alfa de Cronbach) y un análisis factorial exploratorio (mínimos cuadrados generalizados y rotación ortogonal). Para valorar la bondad de ajuste se utilizaron los índices Chi-cuadrado, RMSEA (Root Mean Square Error of Approximation) y el análisis de residuos. Resultados: Se eliminaron los ítems que no cumplieron: el criterio laxo de normalidad (asimetría $<2$ y curtosis $<7$ ), el índice de homogeneidad corregida $>$ .5 y el de comunalidad $>$.5. La escala obtenida, de 14 items, explica un $47 \%$ de la variabilidad, incluyendo 2 factores (Esconder mi dolor y Evitar su dolor) con Chicuadrado/grados de libertad $=1.7$, RMSEA $=0.059 y$ 18\% de residuos; encontrándose diferencias significativas $(\mathrm{p}<.05)$ entre los niveles de formación (Media de bachillerato de 35.38, de FP de 30.88, y de universidad de 27.38). Conclusiones: Se ha conseguido una esta escala breve que mide las actitudes del objetivo $y$ evidenciándose la tendencia de los adultos a evitar que los niños participen del duelo familiar.

Palabras clave: Duelo; Educación para la muerte; Cuestionarios; Información.

\begin{abstract}
Aim: design and validation of a brief scale of adults attitudes towards children in family or collective grief processes. Method: 227 valid questionnaires were obtained; $86 \%$ (191) women and 14\% (31) men, with average age 41 years. From 50 items containing attitudes of adults in situation of grief towards children, an analysis of normality of variables, reliability (alpha of Cronbach) and factor analysis exploratory (generalized least-squares and orthogonal rotation) was performed. To evaluate the goodness of fit, indices Chi-square, RMSEA (Root Mean Square Error of Approximation) andresidue analysis. Results: Items deleted those which did not meet: lax criteria of normality (skewness $<2$ and kurtosis $<7)$, corrected homogeneity index $>.5$ and communality $>.5$. The resulting scale (14 items) explains $47 \%$ of the variability, including 2 factors (Hide my pain and Prevent your pain) with Chi-square/ degrees offreedom $=1.7$, RMSEA $=0.059$ and $18 \%$ of residuals). Significant differences were found $(\mathrm{p}<.05)$ between training levels (Average high school $=35.38$, Vocational training $=30.88$, and University $=27.38)$. Conclusion; A short scale that measures the attitudes of the goal has been obtained and demonstrating the tendency of adults to avoid that children participate in the family duel.
\end{abstract}

Keywords: Grief; Death education; Questionnaires; Information.

Fecha de recepción: 20 de enero de 2016. Fecha de aceptación: 17 de junio de 2016.

Correspondencia sobre este artículo:

E-mail: investigacion@humanizar.es

Dirección postal: Marta Villacieros Durbán. Departamento de Investigación. Centro de Humanización de la Salud. Sector Escultores 39. 28760 Tres Cantos, Madrid, España

(C) 2016 Revista de Psicoterapia 
El duelo se entiende como la reacción normal tras la muerte o pérdida de un ser querido y comprende, en el marco de los componentes actitudinales clásicos cognitivos, afectivos y comportamentales- (Gala, Lupiani y Guillén, 2007) los pensamientos, emociones y comportamientos consecuentes a esta pérdida o a su anticipación (Bonanno y Kaltman, 1999). Se trata de un proceso necesario que permite a la persona la elaboración de la pérdida y la adaptación a la nueva realidad (American Psychiatric Association, 1994).

La mayoría de las personas se recupera en un periodo de tiempo relativamente corto; pero existen factores que pueden condicionar positiva o negativamente este proceso, incrementando o suavizando la intensidad y duración del mismo. Son factores de riesgo, entre otros muchos, el parentesco, la causa del fallecimiento, el tiempo desde la pérdida, la edad, el género, el nivel educativo, las pérdidas significativas previas y la religión (Aries 1974 y 1987; Bermejo, Magaña, Villacieros, Carabias y Serrano, 2011; Gamo y Pazos, 2009; Gala, Lupiani y Díaz, 1991; Raja, Gala, González-Infante, Lupiani, Guillén y Alba, 2002;Urraca, 1982 y 1986; Hilton, 1996; Kastenbaum 1977; Kübler-Ross, 1989).

También se han estudiado como predictores de duelo complicado, en muestras no clínicas, factores como el estilo de afrontamiento, la resiliencia, el bienestar psicológico, el apoyo social percibido, los duelos previos no resueltos y el miedo a la muerte, siendo fundamental el conocimiento de las múltiples variables para la detección precoz de las complicaciones (Bermejo et al., 2011; Gala, Lupiani, Raja, Guillén, González-Infante, Villaverde y Alba, 2002; Villacieros, Magaña, Bermejo, Carabias y Serrano, 2014).

Así mismo, es determinante el concepto de duelo (ideas y creencias) que manejan tanto los dolientes como su entorno, ya que en función de ello se dirige la toma de decisiones o se da una posible evitación del duelo (Amurrio y Limonero 2007). Los estudios en muestras de universitarios españoles (Busquet y Pujol, 2001; Raja, Gala, González-Infante, Lupiani, Guillén y Alba, 2002; Rojas, Muñoz y Alcaraz, 1998; Schmidt, Montoya y García, 2008) apuntan que la formación influye tanto en las actitudes como en las estrategias de afrontamiento. El mismo resultado se encuentra en otros países europeos y estadounidenses (Denton y Wisenbaker, 1977; Wass, 2004).

Los niños a la edad de 8 años ya pueden comprender lo que es la muerte (Sahler, 1983; Urraca, 1985); de hecho, la comprensión de la muerte por parte de los niños y sus actitudes al respecto ha sido objeto de estudios psicológicos desde el primer tercio del siglo pasado (Nagy, 1928; Schilder y Wechler, 1934). Aceptan que todos morimos, incluso ellos (Kane, 1979; Yudkin, 1967). De hecho, se define la primera crisis existencial como ese momento en el que los niños aceptan la universalidad de la muerte y su carácter irreversible (Poch y Herrero, 2003; Santamaría, 2010). Según otros autores, la edad de la comprensión de la muerte se sitúa entre los 9 y 10 años (Nagy, 1948).

Sin embargo, la educación sobre la muerte se ha relegado al ámbito privado, 
donde no siempre se da (Cruz y García, 2007) ya que los padres no se sienten preparados para explicar a sus hijos asuntos relativos a la muerte ni contestar a sus preguntas en torno a ello; según explican ellos mismos, improvisan o evitan hablar del asunto ya que dudan sobre la utilidad de informarles sobre la muerte (TomasSábado y Gómez-Benito, 2004). Por otro lado, en el ámbito escolar se oculta, niega ; e ignora la muerte (Barreto, 2007), ya que tal tema no se incluye como parte de la formación (Herrán y Cortina, 2008; Kreicbergs, Valdimarsdóttir, Onelöv, Henter y Steineck, 2004; Tomás-Sábado y Gómez-Benito, 2004) y en no pocas ocasiones, incluso, de la de los Profesionales Sanitarios en la conocida como "conspiración del silencio" (Gala, Lupiani, Romero, Sánchez-Castillo, Bernalte, Barreto y Lupiani, 2006)

Así, aunque a todos nos afecta este tema, cada vez disponemos de menos estrategias de afrontamiento y nos sentimos más indefensos ante el mismo (Bayés, 2001; Cruz y García, 2007; Gala et al., 2002). Se nos genera miedo, cuando las personas que presentan más miedo a la muerte son también las que presentan más actitudes de evitación, rechazo, estrategias de afrontamiento más ineficaces (Aries, 1974 y 1987; Siracusa et al., 2011; Urraca, 1982; Lonneto y Templer, 1983 y 1988; Ramos y García, 1991) y mayor número de mitos o creencias erróneas sobre el duelo (Amurrio y Limonero, 2007).

Al final, la familia es el principal agente de transmisión de cultura para los niños, por tanto sus actitudes y creencias sobre la muerte influirán de manera decisiva en el posicionamiento y elaboración de situaciones de duelo de los niños (Cruz y García, 2007; Glass y Nahapetyan, 2008). La manera en la que se resuelvan estas situaciones de crisis en la familia determinará, en los niños, la capacidad de afrontar y resolver sus experiencias de pérdida posteriores (Barnes, 1983; Bluebond, 1977; Bela, Razdan, Weller y Weller, 2006; Slaughter y Griffiths, 2007; Villardón, 2001; Viñas y Doménech, 1999; Walsh-Burke, 2006).

A su vez, conocer cómo se construye la comprensión de los niños sobre el tema de la muerte permitirá a los adultos eliminar falsas creencias y manejar mejores estrategias para responder adecuadamente a sus preguntas (Sahler, 1983; Urraca, 1985; Willis, 2002). Dependiendo del punto de desarrollo en el que se encuentre un niño, éste tiene mayor o menor capacidad de comprender la irreversibilidad de la muerte, la finitud, su inevitabilidad y su causalidad. Pero su reacción también depende mucho de las circunstancias externas y de la actitud de los adultos que le rodean (Herrán y Cortina, 2007; Santamaría, 2010).

Según la mayoría de los autores (Herrán y Cortina, 2008; Santamaría, 2010), es importante enfocar la pérdida como una oportunidad de crecimiento con la experiencia. Para ello, se definen (Siracusa, Cruz-Quintana, García-Caro SchmidtRiovalle y Vera, 2011) seis actitudes fundamentales: no mentir, no interrogar, no imponer, no evadirse y no hacer interpretaciones. El corolario de todo ello es que el incluir a los niños en el duelo de los adultos facilita su elaboración a ambas partes.

Las actitudes necesarias para ello incluyen compartir la tristeza para que el 
niño confíe en el adulto como acompañante de su propio duelo;, expresar el dolor para que el niño a su vez abra canales de expresión; y permitir que el niño se apoye en el adulto y reciba la contención necesaria (Tomas-Sábado y Gómez-Benito, 2004). De igual modo el mostrarse a sí mismo tal y como uno se siente en su duelo (en vez de evitar derrumbarse delante del niño), permitir la comunicación fluida (en vez de actuar con frialdad delante del niño), y admitir la perdida sin negar el dolor que existe (en vez de intentar proponer soluciones para salir del dolor) son las estrategias de afrontamiento más adecuadas, ya que al tapar y/o tergiversar la realidad ("el abuelito está dormido", "papá se ha ido de viaje", etc.-en el marco de la "conspiración del silencio" en torno a la muerte, ya aludida) se genera desconfianza en el niño, se le aleja del adulto y, además, al dolor por la pérdida se le suma el dolor por la posibilidad de haber sido abandonado (Tomás-Sábado y GómezBenito, 2004).

Aunque existen escalas sobre el miedo a la muerte, mitos en duelo y actitudes hacia la muerte y percepción sobre la muerte (Amurrio y Limonero, 2007; Boyar, 1964; Robbins, 1991; Siracusa et al., 2011; Templer, 1970), no hemos encontrado ningún instrumento que evalúe y mida específicamente las actitudes de los adultos hacia los niños en este tipo de situación.

Ya que es necesario construir instrumentos de medida que eliminen la subjetividad del evaluador, el objetivo de este estudio ha sido el diseño y validación de una escala de actitudes de los adultos hacia la inclusión de los niños en los procesos de duelo familiares o colectivos.

\section{2- MÉTODO}

\section{1- Participantes}

De una población aproximada de 400 asistentes a las Jornadas de Duelo organizadas por un Centro sociosanitario y formativo de la Comunidad de Madrid, contestaron al cuestionario 231 sujetos (tasa de respuesta aproximada, 57\%) pero se eliminaron 4 cuestionarios por estar incompletos; así, el n final fue de 227 (86\% -191 - mujeres y $14 \%-31$ - hombres), con una media de edad de 41.16 años (DT =14.09, con una edad mínima de 18 y máxima de 75 años).

\section{2- Diseño del instrumento}

Tras una revisión bibliográfica sobre el concepto de duelo, factores que subyacen en él y vivencias sobre el mismo a nivel familiar y de los niños, se elaboró un listado de ítems que respondían a la pregunta Si un familiar cercano (como por ejemplo el abuelo) falleciera ¿cómo lo trataría con sus hijos (u otros niños) entre 8 y 10 años?. Se planteó esa franja de edad ya que-según la mayoría de la literatura revisada al respecto-es cuando los niños pueden comprender los conceptos en torno a la muerte. Losítems, pues, recogían las posibles reacciones actitudinales (cognitivas, afectivas y comportamentales, según lo ya indicado -Gala et al., 2007-) de los 
adultos en posible fase de duelo hacia niños (en iguales circunstancias) con los que ya se podría hablar acerca de la muerte.

Se envió este listado de 72 ítems a distintos Profesionales expertos en duelo y en psicoterapia infantil humanista y sistémica, que lo analizaron en dos fases sucesivas: : En la primera, después de sus comentarios, se decidió eliminar 27 ítems y añadir 5; también se modificaron algunos. En la segunda, se les envió el cuestionario definitivo de 50 ítems, ante el cual mostraron su conformidad.

Las opciones de respuesta a los ítems -directas e inversas-iban del 1 al 5 (de totalmente en desacuerdo a totalmente de acuerdo; según una escala tipo Likert, la más usual para evaluar actitudes -Gala et al. 2007-) . Una vez recodificados los inversos, la presencia de valores altos en las respuestas a la escala global indicant la prevalencia de una actitud de rechazo hacia el duelo familiar o colectivo (y, por tanto, de exclusión de los niños en el duelo familiar).

Además de las variables sociodemográficas, se añadió una pregunta sobre la propia experiencia con niños de 8 a 10 años; Sus opciones de respuesta fueron: a) Tengo (o he tenido) hijos de esa edad, b) Trabajo (o he trabajado) con niños de esa edad, y c) No tengo especial contacto con niños de esa edad. Así mismo, se incluyó un apartado sobre la experiencia en fallecimientos cercanos y la cercanía con el fallecido: ¿Ha sufrido la muerte de algún ser querido en los últimos 2 años?, con las opciones de respuesta: Si/No y El fallecido ¿era para Ud. una persona cercana? puntuables del 1 (nada) al 10 (mucho).

Por último, se añadió una pregunta respecto al miedo a la muerte y su intensidad (¿Le da miedo la muerte? Sí/No y ¿Cuánto miedo le da la muerte?, puntuable también del 1 (nada) al 10 (mucho); ésta se incluyó en aras de utilizarla como variable criterio.

\section{3- Procedimiento}

El cuestionario se ofreció a todos los asistentes a las Jornadas junto con la documentación entregada en las mesas de inscripción, rogándose la colaboración y que lo cumplimentaran antes de finalizar tas dichas Jornadas en las mesas de inscripción.

\section{4- Análisis de los datos}

Se utilizó el paquete estadístico SPSS v20, eliminándose (porque se consideraron incompletos) los ítems con más del $10 \%$ de omisiones; el resto de las omisiones se reemplazaron por la media de la serie. De este modo, con los resultados así obtenidos, se realizó un análisis de normalidad de las variables (prueba de Kolmogorov-Smirnov) y el cálculo de la "Critical Ratio" de los parámetros de asimetría y curtosis (Curran, West y Finch, 1996); también se obtuvieron la medida de adecuación muestral de Kaiser-Meyer-Olkin y la prueba de esfericidad de Bartlett.

Se realizó el análisis de fiabilidad mediante la alfa de Cronbach, eliminándose 
todos aquellos ítems con un índice de homogeneidad corregida y comunalidades menores que .5, así como los valores de saturación menores de .4. Para el Análisis Factorial Exploratorio (AFE) se utilizaron los siguientes métodos: para la elección del número de factores a retener, se tuvieron en cuenta la regla de Kaiser, el método de análisis paralelos y los valores de los índices Chi-cuadrado/grados de libertad $(<2)$, RMSEA (<0.08) y el análisis de residuos (Harman, 1980; Steiger, 2007; Tabachnick y Fidell, 2007). Se eligió el método de mínimos cuadrados generalizados (recomendado para ítems que no siguen la distribución normal) y la rotación ortogonal "varimax" para obtener dimensiones que no se solaparan en sus conceptos.

Para establecer las diferencias de medias entre grupos, se utilizó la $T$ de Student para muestras independientes y el ANOVA de 1 factor $\mathrm{y}$, de igual modo, para establecer las correlaciones el coeficiente $r$ de Pearson.

\section{3- RESULTADOS}

El perfil de los participantes es el de una mujer (191, el 86\%) creyente (157, el 77\%). Aunque la media de edad es de 41 años $(D T=14.09$, mín. de 18, y máx. de 75 años), lo cierto es que no sigue una distribución normal sino que los resultados en esta variable se podrían agrupar en las 3 franjas mayoritarias establecidas en la Tabla 1. Respecto a la formación, la mitad está formada por universitarios (109, $50.9 \%$ ), y, el resto, ha realizado Bachillerato o Formación Profesional (FP). Respecto a las profesiones el 61\% (129) de los participantes está conformado por profesionales sociosanitarios (Tabla 1).

\begin{tabular}{ccrc}
\hline & & Recuento & Porcentaje \\
\hline \multirow{2}{*}{ Sexo } & Mujer & 191 & 86.0 \\
& Hombre & 31 & 14.0 \\
\cline { 2 - 4 } Edad & 18 a 39 & 100 & 44.8 \\
& 40 a 59 & 96 & 43.0 \\
Creencias religiosas & 60 a 75 & 27 & 12.1 \\
\cline { 2 - 4 } Estudios & Creyente & 157 & 77.3 \\
& No creyente & 46 & 22.7 \\
\cline { 2 - 4 } & Bachillerato & 45 & 21.0 \\
Profesión & FP & 60 & 28.0 \\
& Universitario & 109 & 50.9 \\
\cline { 2 - 4 } & Sociosanitaria & 129 & 61.1 \\
Experiencia con niños & No sociosanitaria & 43 & 20.4 \\
de 8-10 años & Estudiante & 39 & 18.5 \\
\cline { 2 - 4 } & Tengo hijos & 84 & 38.9 \\
Fallecimiento en & Trabajo con niños & 75 & 34.7 \\
últimos 2 años & No tengo & 57 & 26.4 \\
\cline { 2 - 4 } & Sí & 126 & 57.3 \\
& No & 94 & 42.7
\end{tabular}




\begin{tabular}{ccrr} 
Cercanía con el fallecido & Baja (1-3) & 5 & 3.8 \\
\cline { 2 - 4 } & Media (4 - 6) & 15 & 11.4 \\
& Alta $(7-10)$ & 112 & 84.8 \\
\cline { 2 - 4 } Miedo a la muerte & Sí & 118 & 53.9 \\
& No & 101 & 46.1 \\
\cline { 2 - 4 } Intensidad del & Baja (1-3) & 72 & 35 \\
miedo a la muerte & Media (4 - 6) & 60 & 29 \\
& Alta $(7-10)$ & 74 & 36 \\
\hline
\end{tabular}

\section{Tabla 1.}

\section{Descripción de la muestra en cuanto a las variables sociodemográficas relativas al objeto de estudio.}

En cuanto a la experiencia con niños de entre 8 y 10 años, los 3 grupos se repartieron de manera equitativa: los que tienen/han tenido hijos de tal edad, los que trabajan/han trabajado con niños de esa edad y los que no han tenido especial contacto con ellos (Tabla 1).

En los últimos dos años, el 57\% (126) tuvo alguna experiencia de fallecimiento de un familiar. Prácticamente la mayoría, el 85\% (112), califica al fallecido como "bastante cercano" (puntuación mayor que 7 en una escala del 1 al 10).

Respecto al miedo a la muerte, la mitad de la muestra $(118,54 \%)$ se inclinó hacia el Sí. La variable criterio, alusiva a la intensidad de miedo a la muerte, se distribuyó de manera platicúrtica, resultando una media poco informativa de 5 (una escala del 1 al 10) y una desviación típica de 2.7; por ello, se dividió a la muestra en 3 franjas de puntuaciones equitativas (Tabla 1).

La distribución de los ítems del cuestionario no resultó normal en las pruebas de normalidad $(p<.05)$. Para realizar el AFE bajo el procedimiento de mínimos cuadrados generalizados se eliminaron 4 ítems que ni tan siquiera cumplían el criterio laxo de "Critical Ratio" de asimetría $<2$ y de curtosis $<7$ (Curran, West y Finch, 1996).

Comprobada la adecuación de los datos $(\mathrm{KMO}=.837$ y prueba de esfericidad de Bartlett significativa $p<.001-)$, se recodificaron las puntuaciones de los ítems inversos y se eliminaron todos aquéllos con índice de homogeneidad corregida y comunalidades menores que .5; quedando, así, los 14 ítems de la forma final del cuestionario.

Según la regla de Kaiser, la escala contenía una estructura de 4 factores mientras que, según, los análisis paralelos, la estructura era unidimensional. Se exploraron por tanto los modelos de entre 1 y 4 factores. Los resultados de bondad de ajuste obtenidos se muestran en la tabla comparativa de modelos (Tabla 2). En ella se observa que todos los índices de ajuste empeoran a medida que se reduce el número de factores en las sucesivas exploraciones. Una vez rotados, se observó que la solución con mejor ajuste (de 4 factores) contenía 2 factores interpretables (de 6 y 4 ítems respectivamente) ya que los 4 ítems restantes resultó que no llegaban a configurar ningún otro factor; así, se decidió mantener esta solución de 2 factores, 
ya que ofrecía mejor equilibrio entre ajuste y parsimonia; de hecho, en ella, solamente dos ítems (15 y 37) obtenían saturaciones menores de .4 (Tabla 3).

\begin{tabular}{|c|c|c|c|c|c|c|c|c|}
\hline \multicolumn{2}{|c|}{ Modelos explorados } & $\mathrm{Chi}^{2}$ & \multirow{2}{*}{$\frac{g l}{41}$} & \multirow{2}{*}{$\frac{p}{.0023}$} & \multirow{2}{*}{$\frac{\text { Chi }^{2} / \mathrm{gl}}{1.7402}$} & \multirow{2}{*}{$\begin{array}{c}\text { RMSEA } \\
.0592\end{array}$} & \multirow{2}{*}{$\begin{array}{c}\% \\
\text { varianza } \\
47 \%\end{array}$} & \multirow{2}{*}{$\begin{array}{c}\begin{array}{c}\% \\
\text { residuos }\end{array} \\
18 \%\end{array}$} \\
\hline 14 ítems & 4 factores & 71.3480 & & & & & & \\
\hline & 3 factores & 100.0000 & 52 & .0001 & 1.9231 & .0661 & $43 \%$ & $26 \%$ \\
\hline & 2 factores & 140.6900 & 64 & .0000 & 2.1983 & .0754 & $37 \%$ & $42 \%$ \\
\hline & 1 factor & 191.9900 & 77 & .0000 & 2.4934 & .0841 & $31 \%$ & $49 \%$ \\
\hline
\end{tabular}

Tabla 2.

Comparación de los índices de ajuste de los modelos factoriales explorados.

El primer factor (6 ítems), que explica un 13\% de la varianza, recoge afirmaciones que hablan sobre el ocultamiento del dolor del adulto. El segundo factor (4 ítems), que explica un 12\% de la varianza, recoge afirmaciones sobre la evitación del dolor a los niños, por ello, se denominaron "Esconder mi dolor" y "Evitar su dolor".

Respecto al análisis de fiabilidad, tanto la escala total $($ alfa $=.849)$ como las subescalas (Tabla 3) mostraron coeficientes alfa de Cronbach elevados.

\begin{tabular}{|c|c|c|c|c|}
\hline \multirow[t]{2}{*}{ Matriz de factores rotados. Escala de 14 ítems } & \multicolumn{4}{|c|}{ Factor } \\
\hline & 1 & 2 & 3 & 4 \\
\hline $\begin{array}{l}\text { 36. Pienso que es mejor mostrarse fuerte ante los niños } \\
\text { 12. Evitaría que me vieran llorar } \\
\text { 16. Es mejor evitarles el dolor a los niños } \\
\text { 32. No quiero que me vean sufrir } \\
\text { 37. Sería dañino para ellos hablar claro sobre el fallecimiento } \\
\text { 15. Retrasaría el momento de contárselo }\end{array}$ & $\begin{array}{l}.595 \\
.508 \\
.506 \\
.489 \\
.399 \\
.372\end{array}$ & $\begin{array}{l}.111 \\
.034 \\
.164 \\
.387 \\
.232 \\
.188\end{array}$ & $\begin{array}{l}.139 \\
.175 \\
.353 \\
.096 \\
.358 \\
.112\end{array}$ & $\begin{array}{l}.136 \\
.249 \\
.290 \\
.174 \\
.102 \\
.284\end{array}$ \\
\hline $\begin{array}{l}\text { 26. Me da miedo hacerles daño hablando de ese tema } \\
\text { 30. Me sentiría culpable del dolor de mis hijos } \\
\text { 33. No se lo diría para que no se sintieran responsables de } \\
\text { mi dolor } \\
\text { 18. Intentaría disimular la ausencia del fallecido }\end{array}$ & $\begin{array}{l}.061 \\
.112 \\
.398 \\
.293\end{array}$ & $\begin{array}{l}.678 \\
.637\end{array}$ & $\begin{array}{l}.027 \\
.198\end{array}$ & $\begin{array}{l}.190 \\
.042\end{array}$ \\
\hline $\begin{array}{l}\text { 43. Si los niños no lo saben no se ven afectados } \\
\text { 44. Utilizaría mentiras piadosas para que no sufran más de } \\
\text { lo necesario }\end{array}$ & .328 & .131 & .935 & .022 \\
\hline $\begin{array}{l}\text { 13. Evitaría que tuvieran que pasar por el trance del funeral o } \\
\text { del entierro } \\
\text { 40R. Invitaría a mis hijos a participar en el funeral }\end{array}$ & $\begin{array}{l}.215 \\
.161 \\
\end{array}$ & $\begin{array}{l}.093 \\
.173 \\
\end{array}$ & $\begin{array}{r}.177 \\
-.025\end{array}$ & $\begin{array}{l}.787 \\
.606\end{array}$ \\
\hline $\begin{array}{r}\text { Alfa de Cronbach } \\
\text { Porcentaje de varianza explicado } \\
\text { Autovalores }\end{array}$ & $\begin{array}{r}745 \\
13 \\
1.8\end{array}$ & $\begin{array}{r}714 \\
12 \\
1.7\end{array}$ & $\begin{array}{r}- \\
11 \\
1.5\end{array}$ & $\begin{array}{r}- \\
10 \\
1.4\end{array}$ \\
\hline
\end{tabular}

$\mathrm{R}$ : ítem cuya puntuación es inversa

Tabla 3.

Matriz de factores rotados (Extracción Mínimos Cuadrados Generalizados, rotación varimax). 
En el análisis de medias de los factores y de la escala ( $T$ de Student o ANOVA de 1 factor) no se encontraron diferencias ni entre sexos, ni entre los 3 grupos de edad definidos, ni entre creencias religiosas (sí/no), ni entre profesiones (sociosanitaria, no sociosanitaria o estudiante), ni entre las personas que tenían experiencia o no con niños, ni entre las personas que han sufrido un fallecimiento en los últimos dos años, ni entre los que dicen tener miedo a la muerte o no. Sin embargo, sí que existen diferencias significativas $(p<.05)$ según el nivel de estudios alcanzados: Conforme aumenta el nivel formativo (bachillerato, FP, universidad) disminuyen las puntuaciones totales obtenidas en la escala $(35,31$ y 27 respectivamente) de manera significativa (Tabla 4).

\begin{tabular}{lrrrrrrr}
\hline & \multicolumn{3}{c}{ Estadísticos descriptivos } & & Bachillerato & FP & Universitario \\
\cline { 2 - 7 } & Media & Mediana & Desv. típ. & Mín.-Máx. & Media & Media & Media \\
\hline Factor 1 & 13,7528 & 14,0 & 4,58068 & $6-29$ & $16,23_{\mathrm{a}}$ & $14,13_{\mathrm{b}}$ & $12,51_{\mathrm{b}}$ \\
Factor 2 & 8,4055 & 8,0 & 3,31482 & $4-20$ & $9,33_{\mathrm{a}}$ & $8,84_{\mathrm{a} . \mathrm{b}}$ & $7,63_{\mathrm{b}}$ \\
TOTAL & 30,1941 & 30,0 & 9,13823 & $14-64$ & $35,38_{\mathrm{a}}$ & $30,88_{\mathrm{b}}$ & $27,38_{\mathrm{c}}$ \\
\hline
\end{tabular}

Nota: Los valores de la misma fila y subtabla que no comparten el mismo subíndice son significativamente diferentes en $p<.05$.

\section{Tabla 4.}

Estadísticos descriptivos de la escala y subescalas resultantes. Diferencias entre las medias de los grupos formados por los distintos niveles formativos (ANOVA de 1 factor).

Respecto a la correlación entre las variables cuantitativas del estudio (Tabla $5)$, se obtienen correlaciones fuertes y significativas entre los factores y la escala total ( $r=.891$ y $r=.797$, ambos con $p<.01)$. La correlación entre ambos factores es de carácter moderado $(r=.544, p<.05)$.

Además, existe una correlación inversa, leve y significativa de la edad con la intensidad del miedo a la muerte $(r=-.196, p<.01)$, con el factor $1(r=-.151, p<$ $.05)$ y con la puntuación total de la escala $(r=-.142, p<.01)$.

Por último, la intensidad del miedo a la muerte correlaciona de manera directa y significativa (aunque leve), con el factor $2(r=.199, p<.01)$.

\begin{tabular}{lcccc}
\hline & Miedo muerte & Factor 1 & Factor 2 & TOTAL \\
\hline Edad & $-.196^{* *}$ & $-.151^{*}$ & -.121 & $-.142^{*}$ \\
Miedo a la muerte & & .032 & $.199^{* *}$ & .114 \\
Factor 1 & & & $.544^{* *}$ & $.891^{* *}$ \\
Factor 2 & & & $.797^{* *}$ \\
\hline
\end{tabular}

* Las correlaciones son estadísticamente significativas en $p<.05$

${ }^{* *}$ Las correlaciones son estadísticamente significativas en $p<.01$

Tabla 5.

Correlaciones de Pearson entre variables del estudio. 


\section{4- DISCUSIÓN}

El diseño del estudio da una respuesta al objetivo habiéndose obtenido una escala fiable y válida, con unos indicadores de bondad de ajuste más que satisfactorios $\left(C h i^{2} / g l<2\right.$ y $\left.R M S E A=0.06\right)$.

A mayor puntuación obtenida en la escala, mayor es el nivel de actitudes desplegadas por el adulto para evitar la inclusión de los niños en el proceso de duelo por un familiar fallecido. Esta escala de 14 ítems se estructura en dos factores, Esconder mi dolor y Evitar su dolor. Este último se incrementa con el miedo a la muerte, mostrándose coherente con la literatura al respecto (Barreto, 2007; Bayés, 2001; Cruz y García, 2007; Gala et al., 2002; Santamaría, 2010, Siracusa et al., 2011).

La actitud evitativa contribuye a disminuir la ansiedad de enfrentarse a una situación dolorosa que no se ha aprendido a manejar. Esconder el propio dolor ayuda a evitar el dolor del niño, ambas actitudes participan del objetivo final de excluir al niño del duelo (Barreto, 2007; Santamaría, 2010), razón que justifica plenamente el hecho de que ambos factores correlacionen.

En la línea de lo que se ha encontrado ya en otros estudios y en diferentes ámbitos (Martí-García et al., 2014), la formación o el nivel de estudios es una variable que se muestra clave en este tipo de situaciones. Cuanto mayor es la formación, menos mitos y creencias erróneas (Amurrio y Limonero, 2007) y también menor evitación del duelo y su expresión (Conesa et al., 2005). Este resultado, dentro del proceso de acumulación de evidencias de validez (American Psychological Association, 1999), apoya la validez de contenido de la escala y confirma que el concepto que se tiene sobre la muerte es clave a la hora de poner en marcha estrategias de afrontamiento de uno u otro tipo (Mc Gorven y Barry, 2000; Schmidt et al., 2008; Williams-Ziegler, 1987).

Los resultados de correlaciones con la variable criterio, aunque son leves, también muestran sentido aportando validez de contenido; a mayor edad, menos miedo a la muerte, por lo que lógicamente se esconde menos el propio dolor (menos se puntúa en el factor 1). Y cuanto más miedo a la muerte más se pretende evitar el dolor a los niños (más se puntúa en el factor 2).

Aporta validez externa al estudio el hecho de que no existan diferencias de puntuación en las comparaciones entre los grupos generados por las variables creencias, profesión, experiencia con niños y miedo a la muerte, ya que amplía el perfil poblacional al que se puede aplicar.

Como limitaciones del estudio podemos mencionar que la dimensionalidad de la escala puede seguir evolucionando. Aunque los pruebas inferenciales $\left(C h i^{2} / g l\right.$ y RMSEA) para el modelo seleccionado son más que satisfactorias, las descriptivas (porcentaje de varianza explicada y análisis de residuos) son moderadas, tenemos 2 ítems son saturaciones menores que las recomendadas (Lloret-Segura, FerreresTraver, Hernández-Baeza, y Tomás-Marco, 2014) y quedan 4 ítems sin interpretar (no incluidos en ningún factor). Futuras investigaciones podrían trabajar sobre este 
resultado. De hecho, diferenciar entre el miedo a la propia muerte o a la muerte del otro podría estar relacionado con los dos factores que constituyen la escala.

\section{5-CONCLUSIONES}

Como corolario, a la vista de lo expuesto, se puede concluir que:

1- Con la metodología expuesta, se ha obtenido una escala valida y fiable que mide las actitudes de los adultos hacia los niños en situaciones de duelo.

2- Esta escala presenta propiedades psicométricas suficientes

3- Dado que tales propiedades se han conseguido con una escala de tan solo 14 ítems, lo que la convierte en una escala breve -con el valor añadido que tal conlleva por su fácil uso- puede ser de gran utilidad para los episodios del duelo en los que la comunicación abierta es difícil y la confusión es elevada.

4- A tenor de los resultados que -en tales episodios y circunstancias- se obtengan, se podrá programar y planificar las estrategias de intervención más adecuadas al respecto en el marco sistémico familiar

\section{Referencias bibliográficas}

American Psychiatric Association (APA) (1994). DSM-IV-TR: Manual diagnóstico y estadístico de los trastornos mentales. Texto revisado. Barcelona: Masson.

American Psychological Association (APA) (1999). Standards for Educational and Psychological Testing. Washington, DC: The American Psychological Association.

Amurrio, L.M., y Limonero, J.T. (2007). El concepto de duelo en estudiantes universitarios. Medicina Paliativa, 14(1), 14-19.

Aries, P (1974). Western attitudes toward death. Baltimore: The Johs Hopkins University Press.

Aries, P (1987). El hombre ante la muerte. Madrid: Taurus

Barreto, M.P., y Soler, M.C. (2007). Muerte y duelo. Madrid: Síntesis.

Barnes, M. (1983). Reacciones de los niños y adolescentes ante la muerte de un padre o hermano. En O. Shaler (Edit.) El niño y la muerte (pp.181-192).Madrid: Alhambra.

Bayés, R. (2001). Psicología del sufrimiento y de la muerte. Barcelona: Martínez Roca.

Bela, A., Razdan, A., Weller, E., y Weller, R. (2006). Children's reactions to parental and sibling death. Current Psychiatry Reports, 8, 115-120.

Bermejo, J.C., Magaña, M., Villacieros, M., Carabias, R., y Serrano, I. (2011). Estrategias de afrontamiento y resiliencia como factores mediadores de duelo complicado. Revista de Psicoterapia, 22(88), 85-95. http:/ /revistadepsicoterapia.com/estrategias-de-afrontamiento-y-resiliencia-como-factores-mediadores-de-duelocomplicado.html

Bluebond, M. (1977). Meanings of death to children. En H. Feifel (Edit.). New Meanings of Death (pp. 91- 116). New York: Thomas Springfield.

Bonanno, G.A. y Kaltman, S. (1999). Toward an integrative perspective on bereavement. Psychological Bulletin, 125(6), 760-77.

Boyar, J. (1964). The construction and partial validation of a scale for measurement of the fear of death. Dissertation Abstracts, 25, 20-21.

Busquet, X., y Pujol, T. (2001). Los estudiantes de enfermería ante la muerte y el morir. Medicina Paliativa, 8, 116119.

Conesa, C., Ríos, A., Ramírez, P., Canteras, M., Rodríguez, M., y Parrilla, P. (2005). Estudio multivariante de los factores psicosociales que influyen en la actitud poblacional hacia la donación de órganos. Nefrología, 25, 684-697. 
Cruz, F., y García, M.P. (2007). SOS... Dejadme morir. Ayudando a aceptar la muerte. Madrid: Pirámide.

Curran, P. J., West, S. G., y Finch, J. F. (1996). The robustness of test statistics to nonnormality and specification error in confirmatory factor analysis. Psychological Methods, 1, 16-29.

Denton, J., y Wisenbaker, M. (1977). Death experiences and death anxiety among nurses and nursing students. Nursing Research, 26, 61-64.

Gala, F.J., Lupiani, M. y Díaz, M. (1991). Sobre las concepciones de la muerte. Rol, 159, 63-66.

Gala, F.J., Lupiani, M. y Guillén, C. (2007). Las actitudes y la conducta interpersonal. En C. Jiménez (Coord.). Ciencias Psicosociales Aplicadas a la Salud (pp.491-511). Córdoba: Servicio de Publicaciones de la Univerisidad de Córdoba.

Gala, F.J., Lupiani, M., Raja, R., Guillén, C., González-Infante, J., Villaverde, C. y Alba, I. (2002). Actitudes psicológicas ante la muerte y el duelo. Una revisión conceptual. Cuadernos de Medicina Forense, 30, 39 50.

Gala, F.J., Lupiani, M., Romero, J.M., Sánchez-Castillo, M. Bernalte, A., Barreto, M. y Lupiani, S. (2006). Communicating Bad News, a challenge for the Clinicians. An approach from the Health Psychology. Journal of Healt Psychology, 18 (2), 77-111.

Gamo, E., y Pazos, P. (2009). El duelo y las etapas de la vida. Revista de la Asociación Española de Neuropsiquiatría, 29(104), 455- 469.

Glass, A., y Nahapetyan, L. (2008). Discussions by elders and adult children about end-of-life preparation and preferences. Preventing Chronic Disease, 5(1), 1-8. Recuperado de http://www.cdc.gov/pcd/issues/2008/ jan/pdf/07_0141.pdf.

Harman, H.H. (1980) Análisis factorial moderno. Madrid: Saltés.

Herrán, A., y Cortina, M. (2007). El "acompañamiento educativo" desde la tutoría en situaciones de duelo. Educación y futuro: revista de investigación aplicada y experiencias educativas, 17, 209-224.

Herrán, A., y Cortina, M. (2008). La educación para la muerte como ámbito formativo: más allá del duelo. Psicooncología, 5, 409-424.

Hilton, J. (1996). Experiencias sobre el morir. Barcelona: Seix Barral.

Kreicbergs, U., Valdimarsdóttir, U., Onelöv, E., Henter, J., y Steineck, G. (2004). Talking about death with children who have severe malignant disease. New England Journal of Medicine, 351, 1175 -1187.

Kübler-Ross, E. (1989). Sobre la muerte y los moribundos. Barcelona: Grijalbo

Lonetto, R. y Templer, D. (1988). La ansiedad ante la muerte. Barcelona: Temis-Edición especial para Zambeletti España, S.A.-

Lloret-Segura, S., Ferreres-Traver, A., Hernández-Baeza, A., y Tomás-Marco, I. (2014). El Análisis Factorial Exploratorio de los Ítems: una guía práctica, revisada y actualizada. Anales de Psicología, 30(3), 1151-1169.

Martí-García, C, García-Caro, M.P., Schmidt-Riovalle, J., Fernández-Alcántara, M., Montoya-Juárez, R., y CruzQuintana, F. (2014). Formación en cuidados paliativos y efecto en la evaluación emocional de imágenes de muerte. Medicina Paliativa, 128. doi: 10.1016/j.medipa.2013.12.007.

Mc Gorven, M., y Barry, M. (2000). Death education: knowledge, attitudes, and perspectives of Irish parents and teachers. Death Studies, 24, 325-33.

Nagy, M. (1928). The child and death. Psychology Stud Univ, 2, 152-157

Nagy, M. (1948). The child's theory concerning death. Journal of Genetic Psychology, 73, 3-27.

Poch, C., y Herrero, O. (2003). La muerte y el duelo en el contexto educativo. Barcelona: Paidós

Raja, R., Gala, F.J., González-Infante, J., Lupiani, M., Guillén, C., Alva, I., (2002). Influencias de las creencias religiosas en las actitudes del Personal Sanitario (P.S.) ante la muerte. Cuadernos de Medicina Forense 29 ( 9), 21-46.

Ramos, F. y García, I. (1991). Miedo y ansiedad ante la muerte. En J. Buendía (Ed.) Psicopatología Clínica y Salud (pp.131-166). Murcia. Servicio de Publicaciones de la Universidad de Murcia.

Robbins, R.A. (1991). Bugen coping with death scale - reliability and further validation. Omega-Journal of Death and Dying, 22(4), 287-299.

Rojas, P., Muñoz, G., y Alcaraz, M. (1998). Características que presentan los alumnos de Enfermería en relación a la muerte. Enfermería Cientifica, 200-201,12-16.

Sahler, S. (1983). El niño y la muerte. Madrid: Alhambra.

Santamaría, C. (2010). El duelo y los niños. Santander: Sal Terrae.

Schmidt, J., Montoya, R., García, M.P., y Cruz, F. (2008). Dos procesos de fin de vida. Cuando la intervención de los profesionales marca la diferencia. Index de Enfermería, 17, 256-260.

Schilder, P. y Wechler, D. (1934). The attitudes of children toward death. Journal of Genetic Psychology, 45, 406451. 
Siracusa, F., Cruz-Quintana, F., Pérez-Marfil, M.N., García-Caro, M.P., Schmidt-Ríovalle, J., y Vera-Martínez, M. (2011). Actitudes y afrontamiento ante la muerte en padres de niños de primaria. Psicología Conductual, $19(3), 627-642$.

Slaughter, V., y Griffiths, M. (2007). Death understanding and fear of death in young children. Clinical Child Psychology and Psychiatry, 12, 525-535.

Steiger, J.H. (2007). Understanding the limitations of global fit assessment in structural equation modeling. Personality and Individual Differences, 42(5), 893-98.

Tabachnick, B.G., y Fidell, L.S. (2007). Using Multivariate Statistics (5 Ed.). New York: Allyn and Bacon.

Templer, D. (1970). The construction and validation of Deaht Anxiety Scale (DAS). Journal of General Psychology, 82, 165-177

Tomás-Sábado, J., y Gómez-Benito, J. (2004). Ansiedad, depresión y obsesión ante la muerte: aproximación conceptual e instrumentos de evaluación. Psicología Conductual, 12, 79-100.

Urraca, S. Actitudes ante la muerte (preocupación, ansiedad, temor) y religiosidad. (Tesis Doctoral). Madrid: Servicio de Publicaciones de la Univ. Complutense.

Urraca, S. (1985). Estudio evolutivo ante la muerte. Jano, 653, 13-14.

Urraca, S. (1986). La muerte en la sociedad contemporánea. En VV. AA. Dilemas éticos de la Medicina actual (pp. 162-179). Madrid: Publicaciones de la Universidad de Comillas

Villardón, L. (2001). El pensamiento de suicidio en la adolescencia. (2 ${ }^{\mathrm{a}}$ Ed.) Bilbao: Universidad de Deusto.

Villacieros, M., Magaña, M., Bermejo, J.C., Carabias, R., y Serrano, I. (2014). Estudio del perfil de una población de personas en duelo complicado que acuden a un centro de escucha de duelo. Medicina Paliativa, 21(3), 91-97.

Viñas, F., y Doménech, E. (1999). El concepto de muerte en un grupo de escolares con ideación suicida. Revista de Psicología General y Aplicada, 52, 89-104.

Walsh-Burke, K. (2006). Grief and loss theories and skills for helping professionals. Boston, MA: Pearson Education.

Wass, H.A. (2004). Perspective on the current state of death education. Death Studies, 28, 289-308.

Willians-Ziegler, J.C. (1987). Cómo aliviar temores comunes. En P. Vilagrasa y P. Peya (Edits.) Paciente terminal y muerte (pp. 156-181). Barcelona: DOYMA.

Willis, C.A. (2002). The grieving process in children: strategies for understanding, educating, and reconciling children's perceptions of death. Early Childhood Education Journal, 29(4), 221-226.

Yudkin, S. (1967). Children and death. Lancet, 1,37-42. 